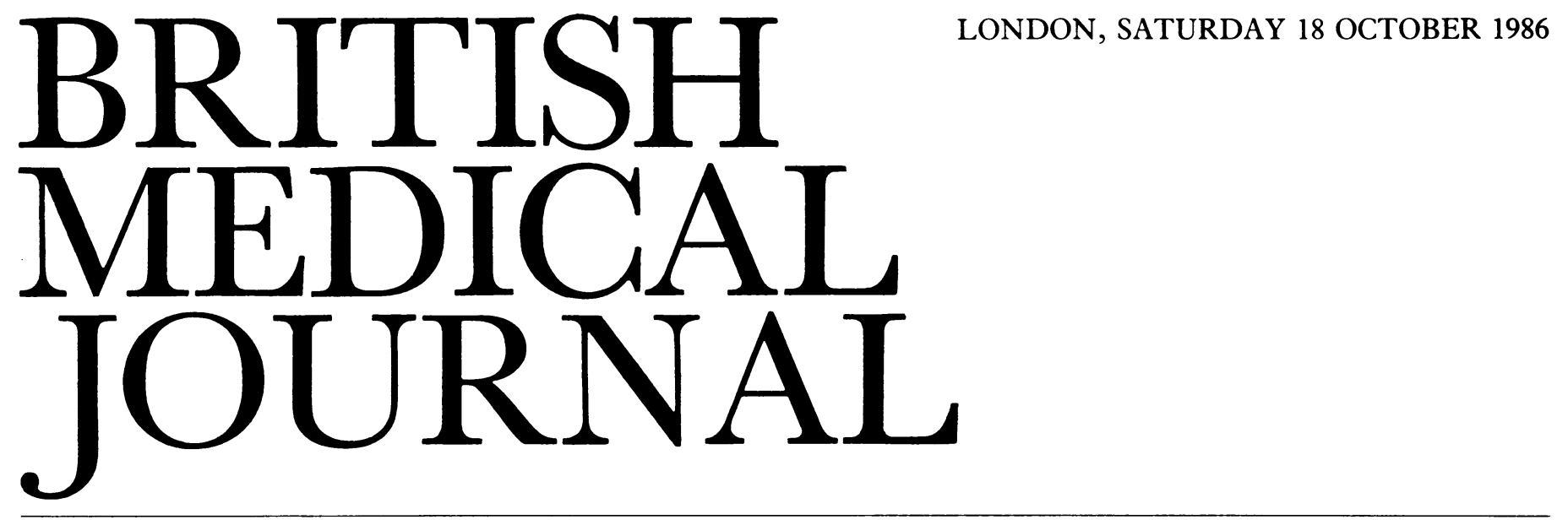

\title{
Alcohol: a new report, but still going backwards
}

Despite increasing evidence of the breadth, depth, and gravity of harm done by alcohol in Britain the message does not seem to be getting through to either the government or the public that something needs to be done. This was shown most clearly by the 1986 budget, in which the tax on alcohol was not increased at all-meaning that in real terms the price has been cut. All the evidence suggests that this will translate into increased consumption and so more alcohol related damage. This failure was worrying in itself, but even more disappointing was the almost complete absence of journalistic comment on the implications for health and society of the Chancellor's decision. Television interviews showed that most people were pleased that the tax had not been increased.

In striking contrast, not only was the tax on tobacco increased above the rate of inflation in the 1986 budget but the Chancellor acknowledged that he was doing this - at least in part-for health reasons. Television interviews in the street showed that even smokers supported the increase. For years the government had refused to use taxation as a means of reducing tobacco consumption, but after each budget in which the Chancellor failed to take action the message would come through strongly that his inaction was bad for health. That this is not the case with alcohol in 1986 means that those campaigning to reduce the harm done by alcohol have a long way to go.

The publication this week of the second report on alcohol from the Royal College of Psychiatrists is another small step forward, but the authors acknowledge that there seems to be a 20 year gap between researchers identifying a problem and possible responses and any use being made of that information. ${ }^{1}$ The Royal College of Physicians (which will itself be producing a report on alcohol early next year) produced its first report on smoking in 1963 and has produced three more since then. Yet still smoking is killing tens of thousands of people and no government has tackled the problem with anything like the drum banging that the present government is using in "fighting" illicit drug abuse.

The tragedy with alcohol is that we seem if anything to be going backwards. The recession is doing its best to keep consumption down, but the government has effectively washed its hands of the problem ${ }^{2}$ - and those campaigning on alcohol are in even more than their customary state of disarray. For those many doctors who cannot keep up with the rapidly changing world of those campaigning to reduce alcohol problems a little history may help.

When the '80s began four groups were trying to reduce alcohol misuse: the National Council on Alcoholism; the Medical Council on Alcoholism; the Federation of Alcoholic Rehabilitation Establishments; and the Alcohol Education Centre. The first three of these shared the same building and yet did not get on with each other. The government, which gave these organisations $£ 300000$ in 1981 , set up a committee to inquire into them-and it found "serious deficiencies.", The committee recommended that they should be scrapped and replaced by one organisation that would be mostly concerned with fostering services for those with alcohol problems. The medical colleges could take over the educational role of the Medical Council on Alcoholism, and the Health Education Council and the Scottish Health Education Group could undertake public education. The inquiry also thought that a small campaigning organisation was necessary - rather on the model of Action on Smoking and Health (ASH).

The government largely accepted these recommendations, and three of the organisations slowly faded away. The Medical Council on Alcoholism refused to die but is less than energetic: it has no direct government grant, and at one stage it had no executive staff at all. Alcohol Concern is the largest organisation, with $£ 500000$ of government money, but by all reports-including some from within the organisation-it is floundering. The ASH style organisation-Action on Alcohol Abuse - has been founded, but unlike ASH it has no government money and thus spends much of its time looking for funding. Finally, there is an Institute of Alcohol Studies, which is funded by the UK Temperance Alliance. Thus four years after the inquiry's report we still have four organisations (two of them directed by people who directed two of the original four), and I strongly suspect that a similar inquiry set up tomorrow would also find "serious deficiencies."

Some of the disarray was shown up by the fiasco of the Alcohol Forum, an organisation in which the drink industry and those concerned about alcohol problems were supposed to meet and work together. The government was keen to encourge the drink industry to spearhead any campaign to reduce alcohol problems. Industry has the money but almost certainly not the will-because, as the Royal College of 
Psychiatrists confirms, the main way to reduce alcohol problems is to contain, and probably reduce, national alcohol consumption. Despite an initial wariness Sir John Crofton, an eminent physician and chairman of the committee that produced a much praised Scottish report on alcohol, 4 agreed to be chairman of the forum. He wanted to test the drink trade's commitment to reducing alcohol problems. He asked the trade to produce a "substantial sum" to fund the forum's work, and the result was that he was promptly sacked. Since then nobody has been "mug enough" to take on chairing this impotent forum, and the last plank of the government's alcohol policy has collapsed.

The Royal College of Psychiatrists makes little or no mention of all this politics in its high minded and clearly written report. But the truth is that much is known about what could be done to reduce alcohol problems, and the report spells out some of those responses. What is lacking is the political will to make the responses and public pressure to make the politicians act-and neither political will nor public pressure is likely to materialise until we have much more effective campaigning organisations.

Assistant Editor,

RICHARD SMITH

AMJ

1 Special Committee of the Royal College of Psychiatrists. Alcohol: our favourite drug. London: Tavistock Publications, 1986

2 Paton A. The politics of alcohol. Br Med f 1985;290:1-2.

3 Department of Health and Social Security. National voluntary organisations and alcohol misuse. London: DHSS, 1982.

4 Scottish Health Education Co-ordinating Committee. Health education in the prevention of alcohol related problems. Edinburgh: Scottish Home and Health Department, 1985.

\section{Treating ovarian cancer}

Ovarian cancer accounts for $6 \%$ of all deaths from cancer in women in Britain and is the commonest cause of death from gynaecological malignancy. Most patients present with tumours extending beyond the pelvis, and the five year survival is inversely related to the stage of the disease as defined by the International Federation of Gynaecology and Obstetrics: for those with stage IIb disease (growth in one or both ovaries with extension to other pelvic tissues) the five year survival is $42 \%$; while for those with stage IV (growth in one or both ovaries with distant metastases, or a pleural effusion containing cancerous cells, or metastases to liver parenchyma) it is $4 \%{ }^{1}$

Radiotherapy, it has been suggested, might "cure" patients with disease up to stage III (growth in one or both ovaries with intraperitoneal metastases outside the pelvis, or tumour in retroperitoneal lymph nodes, or both; or tumour limited to the true pelvis with histologically confirmed malignant extensions to small bowel or omentum). ${ }^{2}$ Other groups, and particularly British ones, have not, however, been able to confirm these results. Patients with more extensive disease have been treated with chemotherapy, usually_until recently_alkylating agents used singly.

The response rates range from $36 \%$ to $65 \%$ (duration 10-14 months), but only a few patients achieve complete remission-and the five year survival is a dismal $0.9 \% .^{3}$

Thus, in an attempt to improve survival, using two or more drugs in combination was a logical step, particularly in view of the success in treating other tumours-notably the lymphomas and Hodgkin's disease. The first study to suggest that this approach improved survival compared a four drug combination (hexamethylmelamine, cyclophosphamide, methotrexate, and fluorouracil) with melphalan. ${ }^{4}$ Improvement was seen only in patients with stage II and III tumours, however, and even these results have not been confirmed by other groups. ${ }^{56}$ Most later drug combinations include cisplatin, and, while toxic, such combinations are highly effective-even for patients with advanced disease.

Are such treatments, though, of long term benefit to the patient? Some controlled studies have shown a survival advantage with cisplatin used in combination ${ }^{7.9}$; others have not. ${ }^{10-13}$ No study, however, has many patients alive after five years irrespective of whether they were showing an improved "survival" at an earlier stage.

So what is the optimum management of patients with stage III and IV ovarian cancer? Can drug combinations containing cisplatin or other toxic drugs be justified, or should we revert to treatment with single agent alkylating agents, particularly for those patients with bulk disease?

The initial surgical management may be crucial. Griffiths was the first to show that the amount of tumour left after an operation was an important prognostic variable. ${ }^{14}$ Survival was uniformly poor if the diameter of the largest residual mass was greater than $1.5 \mathrm{~cm}$ irrespective of tumour volume before resection. ${ }^{14}$ Therefore all patients should have as much tumour as possible resected at the first operation. Also the surgeon should remove the greater omentum and excise, as far as possible, affected lymph nodes. Computed tomography or nuclear magnetic resonance can help identify such nodes. ${ }^{15}$

After surgery patients may be divided into two groups. Those with "minimal residual disease" have by far the better prognosis, and most specialised centres continue to treat such patients with a drug combination containing cisplatin despite its toxicity; this group will probably survive longer with this treatment than if a single agent were used. For the remainder with bulk disease, we think that intensive chemotherapy may still be justified provided there are no contraindications, such as advanced age or poor general health. About half of patients treated with a drug combination will achieve a complete remission, ${ }^{16}$ but relapse is common once treatment is stopped.

Intuitively, we feel that if only there were a means of maintaining the initial remission then a considerable improvement in survival would result. The recent use of monoclonal antibodies has allowed more precise monitoring of treatment, ${ }^{17}$ and such assays could help define the optimum number of treatments, the timing and place of a second laparotomy, and the duration of maintenance treatment. Therefore, such patients should continue to be treated but only in specialised centres with an interest in treatment. Only in this way can trials be conducted to define the optimum treatment and to test new treatments. Ideally all such patients should be included in a clinical trial.

Are there any new drugs on the horizon? The answer is only carboplatin. Whether it will replace cisplatin because of its lower nephrotoxicity is debatable. We think that the response rate will be similar to that with cisplatin, that carboplatin will probably be more myelosuppressive, and that overall survival will not be much improved. Giving platinum intraperitoneally is attractive as it achieves high intracellular drug concentrations in small tumour deposits, and this may be a way forward for those patients with minimal residual disease. . $^{1820}$

Managing advanced ovarian cancer remains difficult, and a simple solution is unlikely to emerge in the foreseeable future. Just as important as concentrating on those who will benefit, however, is that we should not waste resources by 